\title{
Fracture characterization of human cortical bone under mode II loading using the end-notched flexure test
}

\author{
F. G. A. Silva ${ }^{1}$ - M. F. S. F. de Moura ${ }^{2}$ N. Dourado ${ }^{3}$ J. Xavier ${ }^{1,4}$ - F. A. M. Pereira ${ }^{2,4}$. \\ J. J. L. Morais ${ }^{4}$ M. I. R. Dias ${ }^{4}$ P. J. Lourenço ${ }^{5}$ F. M. Judas ${ }^{5}$
}

Received: 2 April 2015 / Accepted: 11 October 2016

(C) International Federation for Medical and Biological Engineering 2016

\begin{abstract}
Fracture characterization of human cortical bone under mode II loading was analyzed using a miniaturized version of the end-notched flexure test. A data reduction scheme based on crack equivalent concept was employed to overcome uncertainties on crack length monitoring during the test. The crack tip shear displacement was experimentally measured using digital image correlation technique to determine the cohesive law that mimics bone fracture behavior under mode II loading. The developed procedure was validated by finite element analysis using cohesive zone modeling considering a trapezoidal with bilinear softening relationship. Experimental load-displacement curves, resistance curves and crack tip shear displacement versus applied displacement were used to validate the numerical procedure. The excellent agreement observed
\end{abstract}

N. Dourado

nunodourado@dem.uminho.pt

1 INEGI - Instituto de Ciência e Inovação em Engenharia Mecânica e Engenharia Industrial, Rua Dr. Roberto Frias, 400, 4200-465 Porto, Portugal

2 Departamento de Engenharia Mecânica, Faculdade de Engenharia da Universidade do Porto, Rua Dr. Roberto Frias, 4200-465 Porto, Portugal

3 CMEMS-UMinho, Departamento de Engenharia Mecânica, Universidade do Minho, Campus de Azurém, 4804-533 Guimarães, Portugal

4 Centre for the Research and Technology of Agro-Environmental and Biological Sciences, CITAB, University of Trás-os-Montes and Alto Douro, UTAD, Quinta de Prados, 5000-801 Vila Real, Portugal

5 Banco de Tecidos Ósseos do Centro Hospitalar e Universitário de Coimbra - CHUC, EPE, Faculdade de Medicina da Universidade de Coimbra, Praceta Prof. Mota Pinto, 3030-396 Coimbra, Portugal between the numerical and experimental results reveals the appropriateness of the proposed test and procedure to characterize human cortical bone fracture under mode II loading. The proposed methodology can be viewed as a novel valuable tool to be used in parametric and methodical clinical studies regarding features (e.g., age, diseases, drugs) influencing bone shear fracture under mode II loading.

Keywords Bone · Fracture characterization · Mode II · ENF test $\cdot$ Cohesive zone modeling

\section{Introduction}

Bone fracture characterization is becoming a very prominent research topic mainly due to aging of population and the consequent increase in age-related diseases. The main goal is to contribute to the improvement of medical prevention and treatment leading to increase the life quality of patients. In the last decade there have been several works dedicated to fracture behavior of bone namely development of finite element-based models to predict bone fracture [5]. The large majority is about fracture characterization under mode I loading [7-9, 23]. However, during daily activities of humans shear fractures are prone to occur. A common example is the case of sudden twisting movements during sport activities that lead to frequent fractures under an almost pure shear loading. Consequently, bone fracture characterization under mode II loading is particularly relevant although only few studies [13] address this topic. The compact shear test was proposed by Norman et al. [10] and Brown et al. [1] for mode II fracture characterization of human cortical bone and used it to evaluate fracture toughness dependency on bone location and age. However, several drawbacks can be pointed to this test as is the case of 
insensibility of specimen compliance as a function of crack length making difficult the execution of compliance calibration, the existence of some mode mixity instead of the intended pure mode II and unstable crack growth. Zimmermann et al. [24] proposed the asymmetric four-point bending test considering specimens with a pre-crack located at the specimen mid-span for tests under mixed-mode I+II loading. The pure mode II loading case is achieved when the pre-crack is aligned with the center of loading device which corresponds to null bending moment, only remaining shear loading. However, numerical simulations performed by Pereira et al. [12] revealed the existence of mode I loading in the fracture process zone, thus making the test inadequate for a pure mode II fracture characterization. Recently, miniaturized versions of the end-notched flexure (ENF) and end-loaded split (ELS) tests were proposed [3, 12] for fracture characterization under mode II loading of bovine cortical bone tissue. It was verified that both tests can be used in this context, when a cautious choice of specimen dimensions is made. In fact, self-similar crack growth should be assured even for a small crack extent to provide a valid evaluation of fracture energy.

In this work, the application of the miniaturized version of the ENF test to human cortical bone fracture characterization under mode II loading is performed. Two main objectives are envisaged. The first one is to verify whether the ENF test is appropriate for fracture characterization of human cortical bone under mode II loading. Previous studies on bovine bone revealed limitations on specimen dimensions possible to get in the diaphysis region. An advantage of human bone relative to bovine is the available length to produce specimens. Though bovine bones are longer than the human's, they present a more pronounced curvature of the diaphysis which leads to shorter planar specimens. This aspect is relevant concerning the production of ENF specimens since the available region for selfsimilar crack growth in this test is quite limited. The second objective addresses the development of a direct method to evaluate the cohesive law (CL) characterizing fracture of human cortical bone under mode II loading. In the course of the experimental ENF tests, the crack tip shear displacement was monitored by digital image correlation (DIC) and subsequently combined with strain energy release rate to get the CL. This law is representative of the material fracture process and its evaluation is important for the assessment of several aspects influencing bone fracture behavior. Numerical analysis with cohesive zone modeling was also performed to validate the procedure. Excellent agreement was obtained in the global and local parameters involved in all the procedure, thus making this test a suitable choice for studies involving fracture characterization of human cortical bone under mode II loading. This is a relevant novelty regarding the implementation of methodical studies concerning endogenous and exogenous aspects influencing bone health.

\section{Methods}

\subsection{Specimen preparation}

For the present work, cortical bone from two tibias of different young male donors (donor A was 21 and donor B 32 years old) were used. Five ENF specimens (two from donor A and three from donor B) were prepared from the medial region of the tibia (Fig. 1a), in the diaphysis. Owing to restrictions related to tibia curvature in the diaphysis region, the available length was about $85-90 \mathrm{~mm}$. After machining and milling operations involving the elimination of endosteal and periosteal tissues, the specimen nominal dimensions (see Fig. $1 \mathrm{~b}$ and c) were: $2 L=80 ; 2 h=6$; $B=2.5$ (units: $\mathrm{mm}$ ). Specimens were continuously irrigated with physiological saline at all steps of the machining process and then frozen at $-20{ }^{\circ} \mathrm{C}$ for storage before testing. The initial crack length $a_{0}$ was introduced in two steps: Initially, a circular saw was used to produce a notch of thickness $0.3 \mathrm{~mm}$ and length $20 \mathrm{~mm}$; subsequently, a pre-crack of about $0.5 \mathrm{~mm}$ was induced considering a sharp blade inserted into the notch, rendering $a_{0}=21 \mathrm{~mm}$. This last operation was executed in the test machine by moving the actuator $0.5 \mathrm{~mm}$ with the blade positioned at the notch tip with a velocity of $100 \mathrm{~mm} / \mathrm{s}$. The pre-cracks were made in the central region of the diaphysis for each pair of specimens harvested from the same tibia. This procedure provides fracture in the same region for the two specimens, thus diminishing the scatter of the mechanical response.

A previous work of the research group concerning the application of the ENF test [3] on fracture characterization of bovine bone has revealed that the crack tends to deviate from its initial mid-plane. This can be viewed as a negative aspect since it leads to mixed-mode I+II loading, which precludes a truthful characterization of bone fracture under mode II loading. To overcome this difficulty, two longitudinal V-shaped grooves $\left(60^{\circ}\right)$ with a depth of $0.4 \mathrm{~mm}$ were manufactured in order to induce crack propagation along the specimen mid-plane. Hence, the width of resistant section (i.e., $b=B-2 \times 0.4=1.7 \mathrm{~mm}$ in Fig. 1c) becomes smaller relative to the one of the specimen arms which is taken into account in the determination of fracture energy.

\subsection{Experimental tests}

The ENF tests (Fig. 2a) were carried out in a servo-electrical testing system (MicroTester INSTRON ${ }^{\circledR}$ 5848) with a load cell with the capacity of $2 \mathrm{kN}$ considering a constant displacement rate of $0.5 \mathrm{~mm} / \mathrm{min}$ and an acquisition 
Fig. 1 a Segment of a tibia showing the specimen source, b schematic representation of the ENF test with its orthotropic directions (L-longitudinal and $\mathrm{T}$ - tangential) and $\mathbf{c}$ lateral view of the specimen showing the lateral grooves

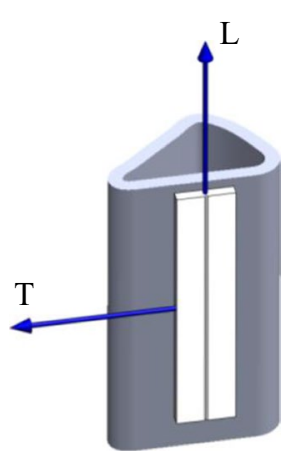

(a)

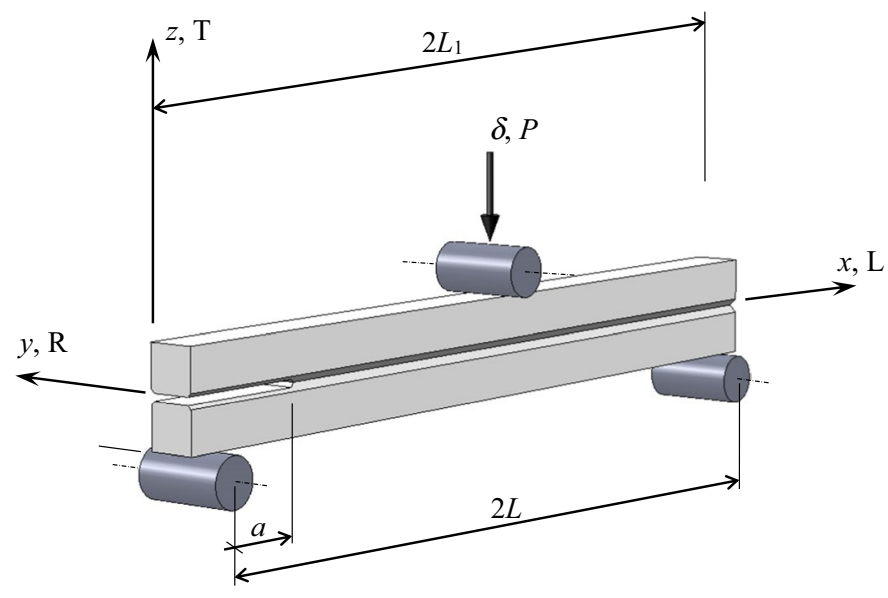

(b)

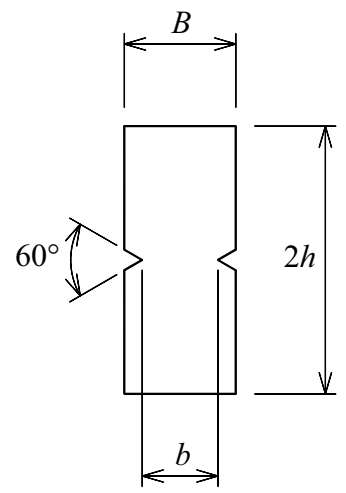

(c)

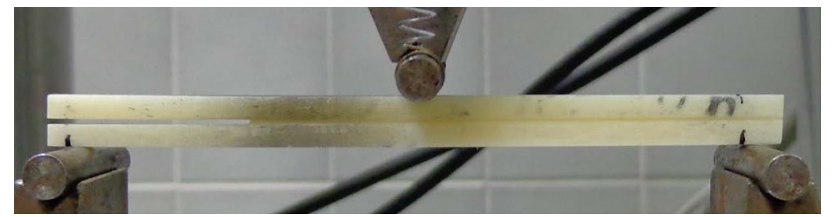

(a)

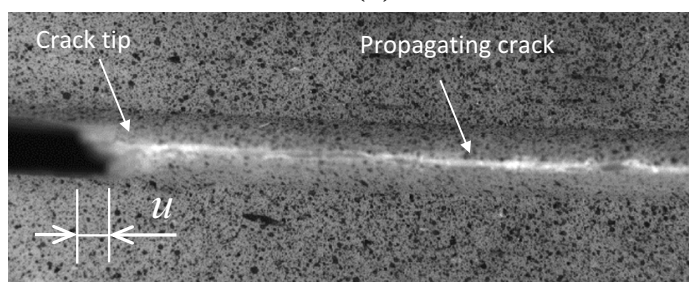

(b)

Fig. 2 a Setup of the ENF test; $\mathbf{b}$ detail of the crack tip under mode II loading

frequency of $5 \mathrm{~Hz}$. The specimens were loaded in displacement control under typical environmental laboratorial conditions $\left(65 \%\right.$ of relative humidity at $\left.20{ }^{\circ} \mathrm{C}\right)$. Three cylinders of $5 \mathrm{~mm}$ diameter were used as supports and loading device. Two lubricated Teflon ${ }^{\circledR}$ films were placed in the pre-crack zone between the specimen arms (region of length $a$ in Fig. 1b) in order to minimize friction effects induced by relative sliding of specimen arms at this region. Five ENF specimens were tested. However, one of them (from donor A) has broken before crack propagation under mode II loading, which led to four valid results. The corresponding load-displacement curves ( $P-\delta$ curves) were recorded and used in the equivalent crack length data reduction scheme to assess the resistance curves ( $R$-curves) under mode II loading. In addition, the crack tip shear displacement (CTSD) was measured by digital image correlation (DIC). The objective is to evaluate experimentally the cohesive laws (CL) characterizing fracture of human bone under mode II loading.

\subsection{Digital image correlation (DIC)}

DIC is an imaged-based technique which provides the measurement of displacements on subregions defined across images of a target object under deformation [11, 20, 22]. The object surface of interest must be locally characterized by a textured pattern with suitable contrast, isotropy and average size. At the tissue level, this pattern was 
Fig. 3 Mesh used in the numerical simulations

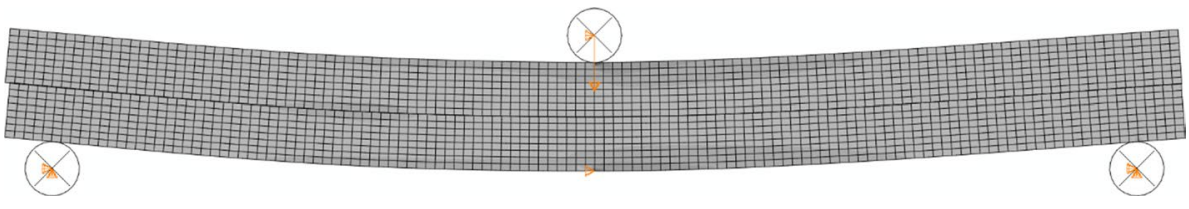

created by means of airbrush painting, by randomly spreading black paint over the bright natural surface of cortical bone. A qualitative visualization of the painted specimens is shown in Fig. 2a, b. In this work, the ARAMIS DIC2D system was used [19]. A charge-coupled device (CCD) camera was coupled with a telecentric lens aiming a region of interest of $7.1 \times 5.4 \mathrm{~mm}^{2}$. This level of magnification was deemed suitable for observing the crack initiation and propagation for the purposes of this study, in a balance between field of view and required spatial resolution. Moreover, this type of lens has the advantage of being less sensitive to out-of-plane parasitic movement within its range of depth of field. The lighting, aperture and shutter time were then set in order to maximize the pixel gray distribution over the dynamic range of the camera sensor, avoiding both under- and over-saturation. DIC measurement parameters must be carefully chosen in a balance between accuracy (large subsets) and spatial resolution (small subsets). In this work, the size of subsets were defined by $15 \times 15$ pixel $^{2}$ $\left(0.060 \times 0.060 \mathrm{~mm}^{2}\right)$ with a slightly overlapping among subsets with step of $13 \times 13$ pixel $^{2}\left(0.052 \times 0.0524 \mathrm{~mm}^{2}\right)$ for enhancing spatial resolution. Sequence of images, taken during simple translation rigid-body movements, were systematically taken before testing to check image quality and accuracy by comparing measurement with reference theoretical deformation $[16,17]$. The displacement resolution, measured as the standard deviation over Gaussian noisy maps obtained by subtracting the average value over fullfield displacements on translated images, was of the order of $1-2 \times 10^{-2}$ pixel $(0.04-0.08 \mu \mathrm{m})$.

\subsection{Numerical analysis}

The ENF tests were simulated in Abaqus ${ }^{\circledR}$ software considering 2016 eight-node plane stress elements (width $B$ ) with 126 six-node cohesive elements (width $b$ ) positioned at the specimen mid-plane to simulate the fracture process. A developed user subroutine was used to implement the trapezoidal with bilinear softening cohesive law [15]. The pre-crack was simulated considering "opened" cohesive elements, i.e., elements that only transmit normal compressive stresses to avoid interpenetration between specimen arms. The boundary conditions were imposed according to the experiments (Fig. 3). A nonlinear geometrical analysis was adopted. The load was applied under displacement control considering small increments $(0.5 \%$ of the applied displacement) to provide smooth damage propagation during loading. The following elastic properties $\left(E_{\mathrm{L}}=13.6 ; E_{\mathrm{T}}=6.0 ; G_{\mathrm{LT}}=3.28\right.$ all in $\mathrm{GPa}$ and $v$ $\mathrm{LT}=0.3)$ presented in Ref. [18] were used in the numerical simulations.

One of the advantages of the CZM models is that they converge to a solution on the condition that at least 3 or 4 integration points are under softening simultaneously along the crack path [4]. This condition was largely satisfied in the present analyses.

\subsection{Cohesive laws}

The objective is to provide a suitable methodology allowing to determine the cohesive law (CL) that characterizes human bone fracture under mode II loading using the ENF test. The direct method is based on measurement of the crack tip shear displacement (CTSD, here represented in equations by $u$; see Fig. $2 \mathrm{~b}$ ) at the crack tip and on the evaluation of the strain energy release rate under mode II loading. The cohesive law $(\tau=\mathrm{f}(u))$ can be obtained from differentiation of the following equation [6],

$G_{\mathrm{II}}=\int_{o}^{u} \tau(\bar{u}) \mathrm{d} \bar{u}$

where $G_{\mathrm{II}}$ is the strain energy release rate under mode II loading and $\bar{u}$ the shear relative displacement varying from zero up to the current value $u$, which yields

$\tau(u)=\frac{d G_{\mathrm{II}}}{d u}$.

This equation requires the determination of the evolution of the strain energy release rate in the course of the ENF test. Classical methods frequently used to measure the evolution of the strain energy release rate are based on crack length measurement during the test. However, as discussed in [3] and also observed in the present work, the crack length monitoring in the course of the test is quite difficult to perform, owing to non-negligible fracture process zone that develops ahead of the crack tip. In this context, an equivalent crack length procedure based on beam theory and specimen compliance can be used. Considering the Timoshenko beam theory, the specimen compliance $C=\delta / P$, being $\delta$ the applied displacement and $P$ the resulting load (Fig. 1b), becomes

$C=\frac{3 a^{3}+2 L^{3}}{8 E_{\mathrm{L}} B h^{3}}+\frac{3 L}{10 G_{\mathrm{LT}} B h}$ 
where specimen dimensions are identified in Fig. 1b, c and $E_{\mathrm{L}}$ and $G_{\mathrm{LT}}$ are the elastic longitudinal and shear moduli, respectively. In order to account for variability of elastic properties between specimens and stress concentration at the crack tip, the initial values of compliance $\left(C_{0}\right.$ in $\left.\mathrm{mm} / \mathrm{N}\right)$ obtained from the initial slope of load-displacement curve and crack length $\left(a_{0}\right)$ were used in the previous equation to assess an equivalent elastic modulus

$E_{\mathrm{f}}=\frac{3 a_{0}^{3}+2 L^{3}}{8 B h^{3}}\left(C_{0}-\frac{3 L}{10 G_{\mathrm{LT}} B h}\right)^{-1}$.

It has been observed that the influence of the shear modulus $G_{\mathrm{LT}}$ is quite small and a typical value can be used without altering significantly the results. Following this procedure, the elastic modulus $E_{\mathrm{L}}$ is replaced by the equivalent one $E_{\mathrm{f}}$ in the equations used to estimate the fracture toughness, thus mitigating the influence of scatter of elastic properties on $G_{\mathrm{II}}$. During propagation, an equivalent crack length can be estimated from Eq. (3) and including the result of Eq. (4)

$a_{e}=\left[\frac{C_{c}}{C_{0 c}} a_{0}^{3}+\frac{2}{3}\left(\frac{C_{c}}{C_{0 c}}-1\right) L^{3}\right]^{1 / 3}$

where

$C_{\mathrm{c}}=C-\frac{3 L}{10 B h G_{\mathrm{LT}}} \quad$ and $\quad C_{0 \mathrm{c}}=C_{0}-\frac{3 L}{10 B h G_{\mathrm{LT}}}$.

The evolution of $G_{\text {II }}=\mathrm{f}\left(a_{\mathrm{e}}\right)$ can easily be obtained combining the Irwin-Kies equation

$G_{\mathrm{II}}=\frac{P^{2}}{2 B} \frac{d C}{d a}$.

with Eq. (3) yielding

$G_{\mathrm{II}}=\frac{9 P^{2} a_{\mathrm{e}}^{2}}{16 B^{2} h^{3} E_{\mathrm{f}}}$.

This procedure provides the Resistance-curve ( $R$-curve) which depicts the evolution of $G_{\mathrm{II}}=\mathrm{f}\left(a_{\mathrm{e}}\right)$ in the course of the test using exclusively the applied load-displacement data.

With the purpose of getting the CL, it is necessary to establish the relation of $G_{\text {II }}$ with the CTSD measured by DIC. The relationship $G_{\text {II }}=\mathrm{f}(u)$ must then be differentiated to obtain the CL according to Eq. (2). Since measurement noise is prone to be amplified by numerical differentiation, experimental data are fitted using a suitable function approximation to smoothen noise data before differentiation. Attending to a theoretical knowledge of the shape of the $G_{\text {II }}=\mathrm{f}(u)$ function $[2,14,21,22]$, a suitable trial function can be used in least-squares regression, such as the logistic function [20, 22]. However, in order to hold the

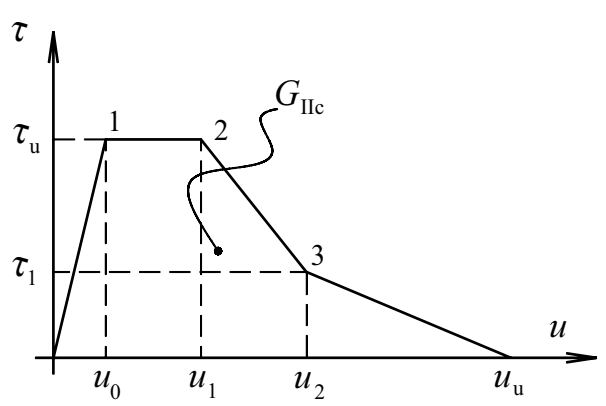

Fig. 4 Numerical trapezoidal with bilinear softening cohesive law

unconstrained problem of not assuming a priori the shape of the cohesive law, in this study a smooth spline approximation scheme was implemented instead $[14,21]$. In this case, a smooth spline $s$ is reconstructed which minimizes the following objective function [21]

$E(p)=p \sum_{i}\left(y_{i}-s\left(x_{i}\right)\right)^{2}+(1-p) \int\left(\frac{d^{2} s}{\mathrm{~d} x^{2}}\right)^{2} \mathrm{~d} x$

where $\left(x_{i}, y_{i}\right)$ represent the data points and $p$ the specified smoothing parameter defined in the range of 0 up to 1 . In Eq. (9), it should be noticed that the first term corresponds to the well-known mean squared error of assuming curve $s(x)$ to estimate $y$. A simple least-squares straight-line fitting is achieved by choosing $p=0$, while a piecewise cubic spline interpolation (passing through all the data points) is performed when $p=1$. Therefore, the parameter $p$ must be chosen with regard to the degree of required data smoothing.

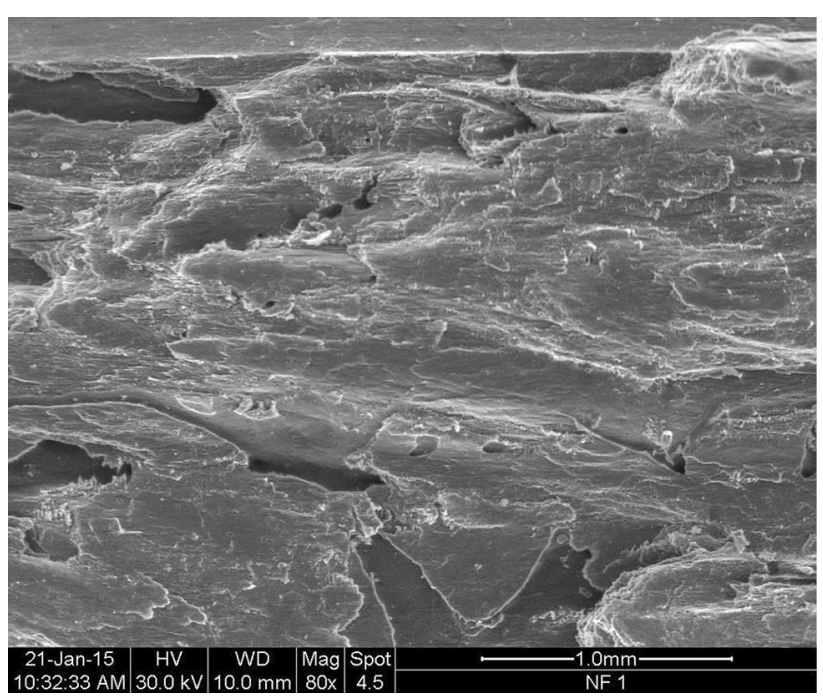

Fig. 5 Microscope photography of the fractured surface of human cortical bone under mode II loading 

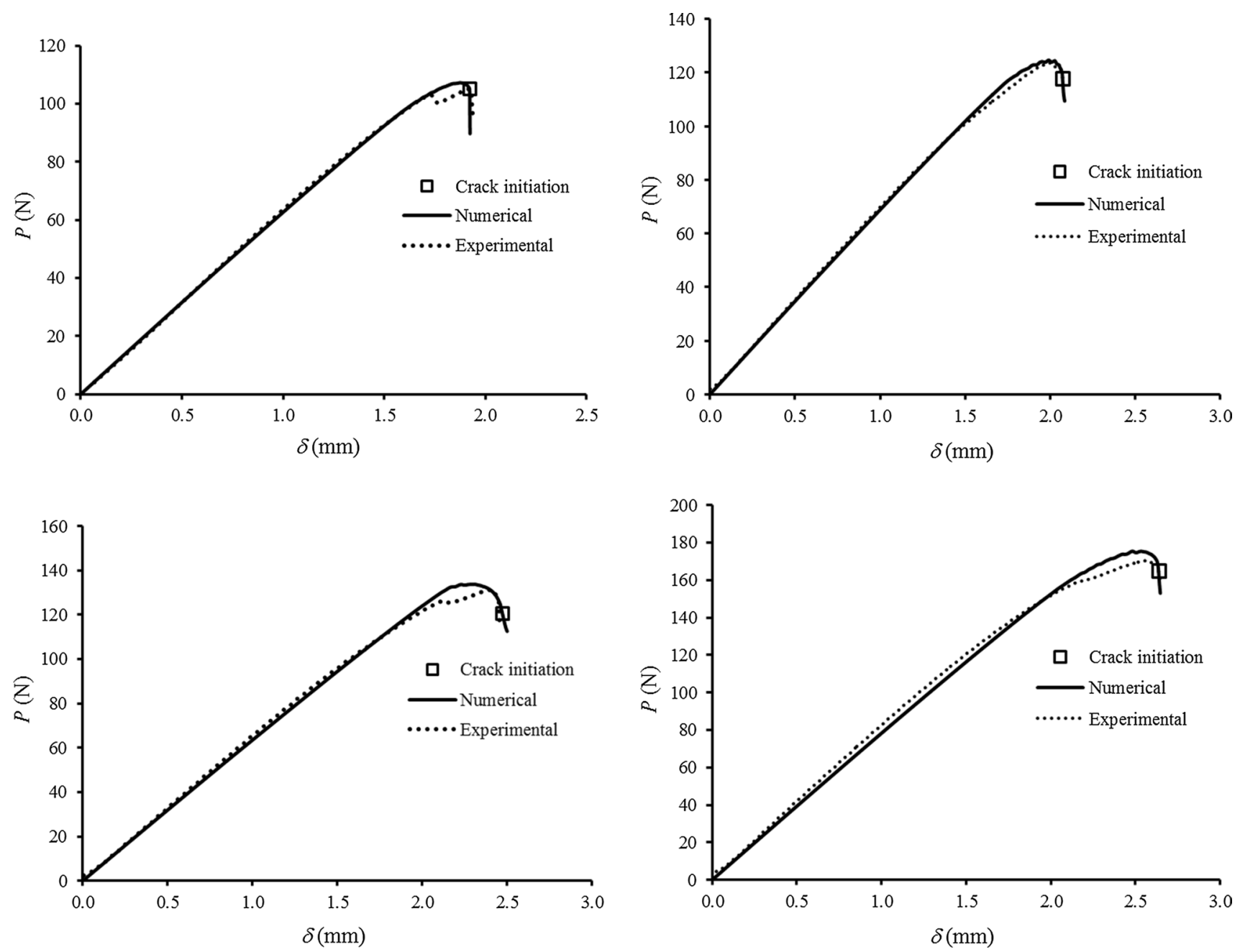

Fig. 6 Experimental and numerical load-displacement curves of the ENF tests

\subsection{Trapezoidal with bilinear softening $C L$}

Taking into account the shape of the CL obtained experimentally, it was decided to adjust trapezoidal with bilinear softening laws (Fig. 4) to be used in finite element analysis performed for the purpose of procedure validation. The cohesive zone model used in this work was previously developed in [15]. For the sake of clarity, the main equations are summarized below.

In the initial linear ascending branch, i.e., for $u<u_{0}$, the following relation is valid

$\tau(u)=k u$

where $k$ is the interface stiffness. When the local strength $\tau_{\mathrm{u}}$ is attained, damage onset takes place and stiffness decreases which is described by the following relationship

$\tau(u)=(1-d) k u$ where $d$ is the damage parameter ranging between $0\left(u=u_{0}\right)$ and $1\left(u=u_{u}\right)$. In the plateau region (for $u_{0} \leq u \leq u_{1}$ ) a slighter decrease in stiffness in the vicinity of local strength $\tau_{\mathrm{u}}$ is simulated and the damage parameter is given by

$d=1-\frac{u_{0}}{u}$.

In the first linear descending branch of the bilinear softening (for $u_{1} \leq u \leq u_{2}$ ), the damage parameter becomes

$d=1-\frac{\frac{\sigma_{2}}{k}\left(u-u_{1}\right)+u_{0}\left(u_{2}-u\right)}{u\left(u_{2}-u_{1}\right)}$

while in the second one (for $u_{2} \leq u \leq u_{\mathrm{u}}$ ),

$d=1-\frac{\sigma_{2}\left(u_{\mathrm{u}}-u\right)}{k u\left(u_{\mathrm{u}}-u_{2}\right)}$. 
The coordinates of the inflection points (1-3) of Fig. 4 were obtained by adjusting the numerical CL to the experimental one. The main goal is to verify whether the numerical load-displacement curve resulting from the ENF simulation agrees with the experimental one.

\section{Results and discussion}

\subsection{Experimental}

A detail of the crack tip region obtained by the optical system after damage development under mode II loading is presented in Fig. 2b. A shear displacement at the crack tip without any evidence of opening displacements (mode I component) is clearly visible, which is an indication of an almost pure mode II loading, as envisioned. However, a slight tortuous crack path can be observed which constitutes part of the complex damaging mechanism developed
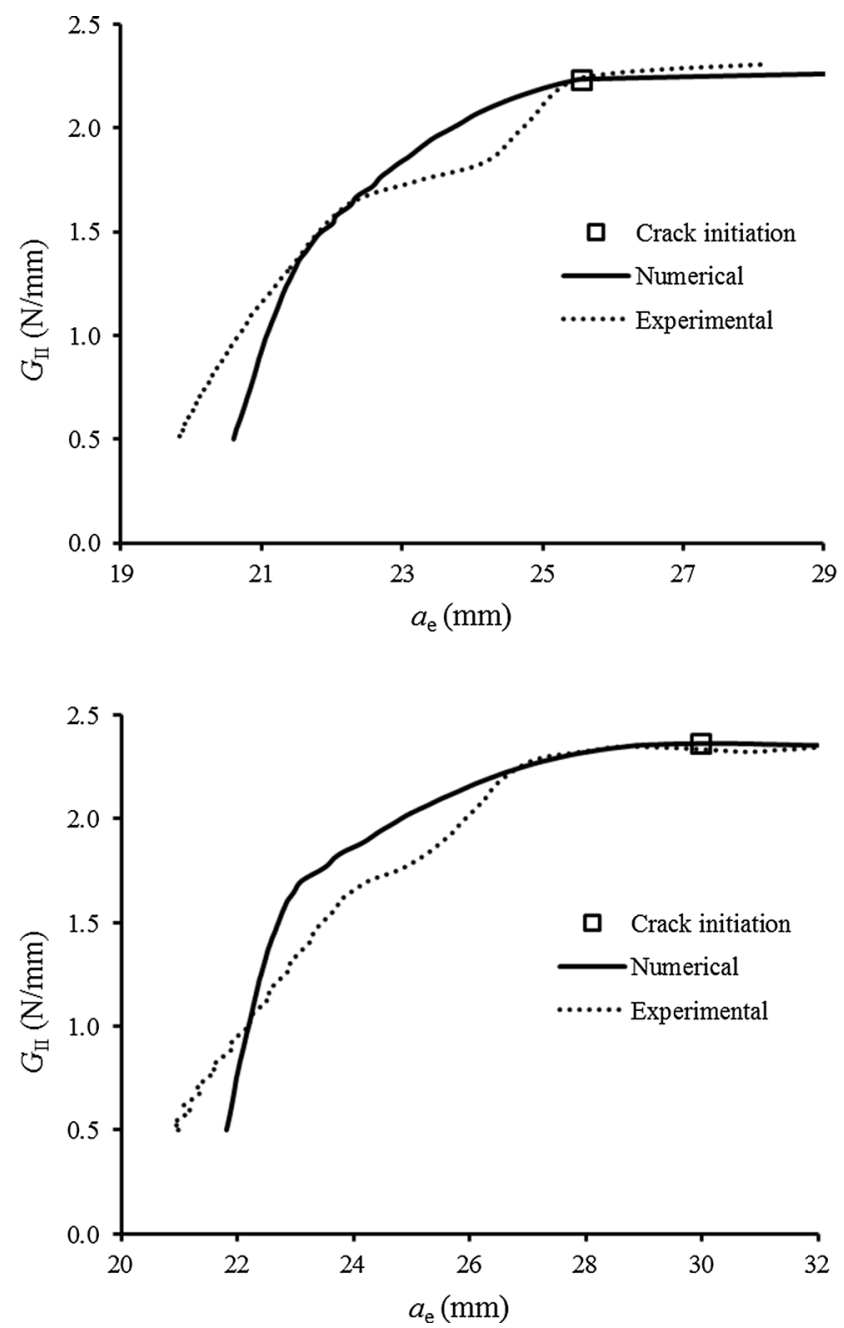

during damage growth. It should also be noted the difficulty on crack tip detection, since crack grows with their faces in contact.

Figure 5 shows the fractured surface under mode II loading. A complex and irregular surface is visible which constitutes a source of stress concentrations that contribute to the slight winding crack path. Evidences of shear failure are visible in longitudinal aligned fractured microstructures and absence of pullout in the direction normal to the fractured plane. These features are particularly relevant, meaning that local mode I component is negligible, i.e., an almost pure mode II loading is taking place as envisioned. The surface texture is quite irregular leading to dissipation of a non-negligible amount of energy due to friction effects. These aspects (shear failure of microstructures and friction effects) constitute the fundamental damage mechanism of cortical bone under pure mode II loading. Both contribute to increase the energy being dissipated in the so-called fracture process zone (FPZ), making fracture of
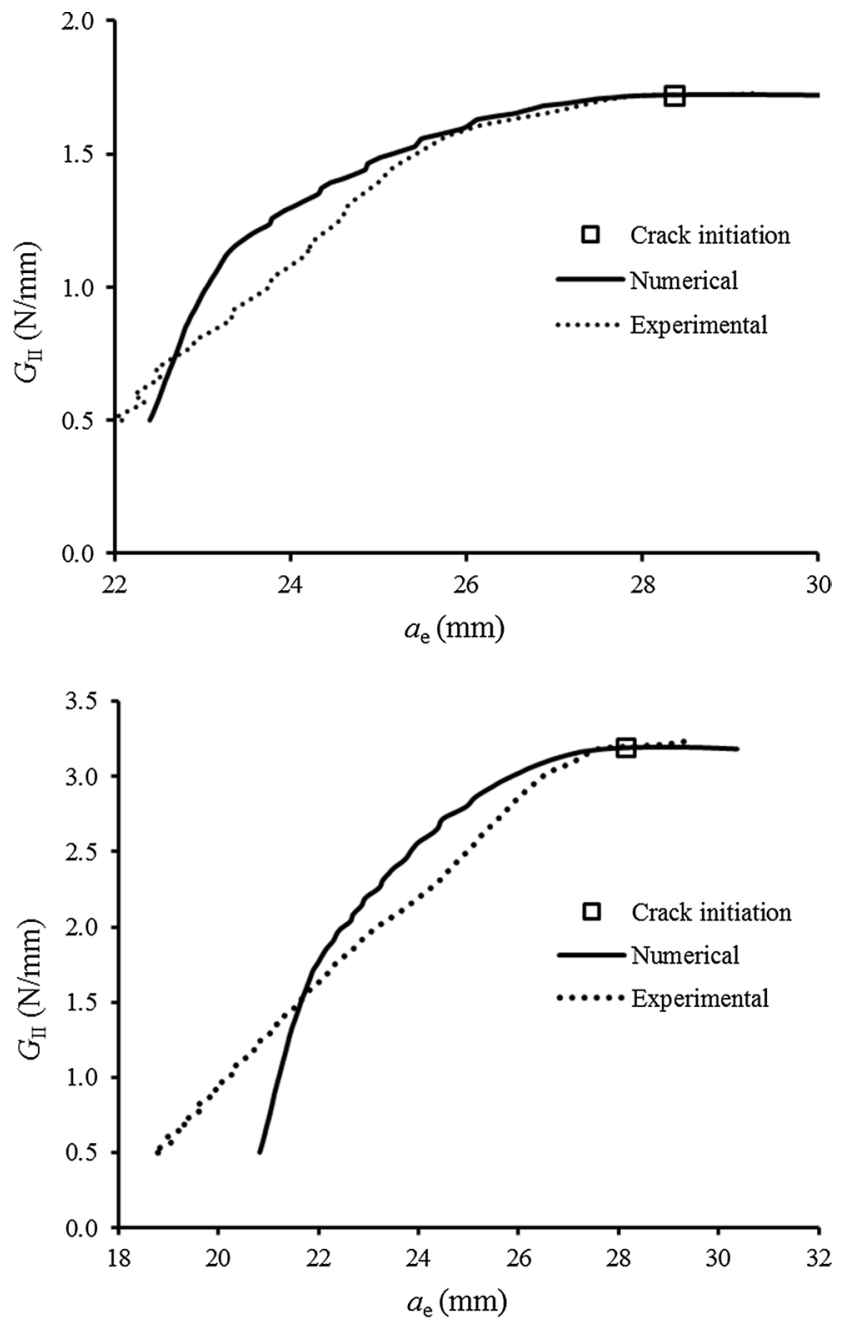

Fig. 7 Experimental and numerical $R$-curves of the ENF tests 

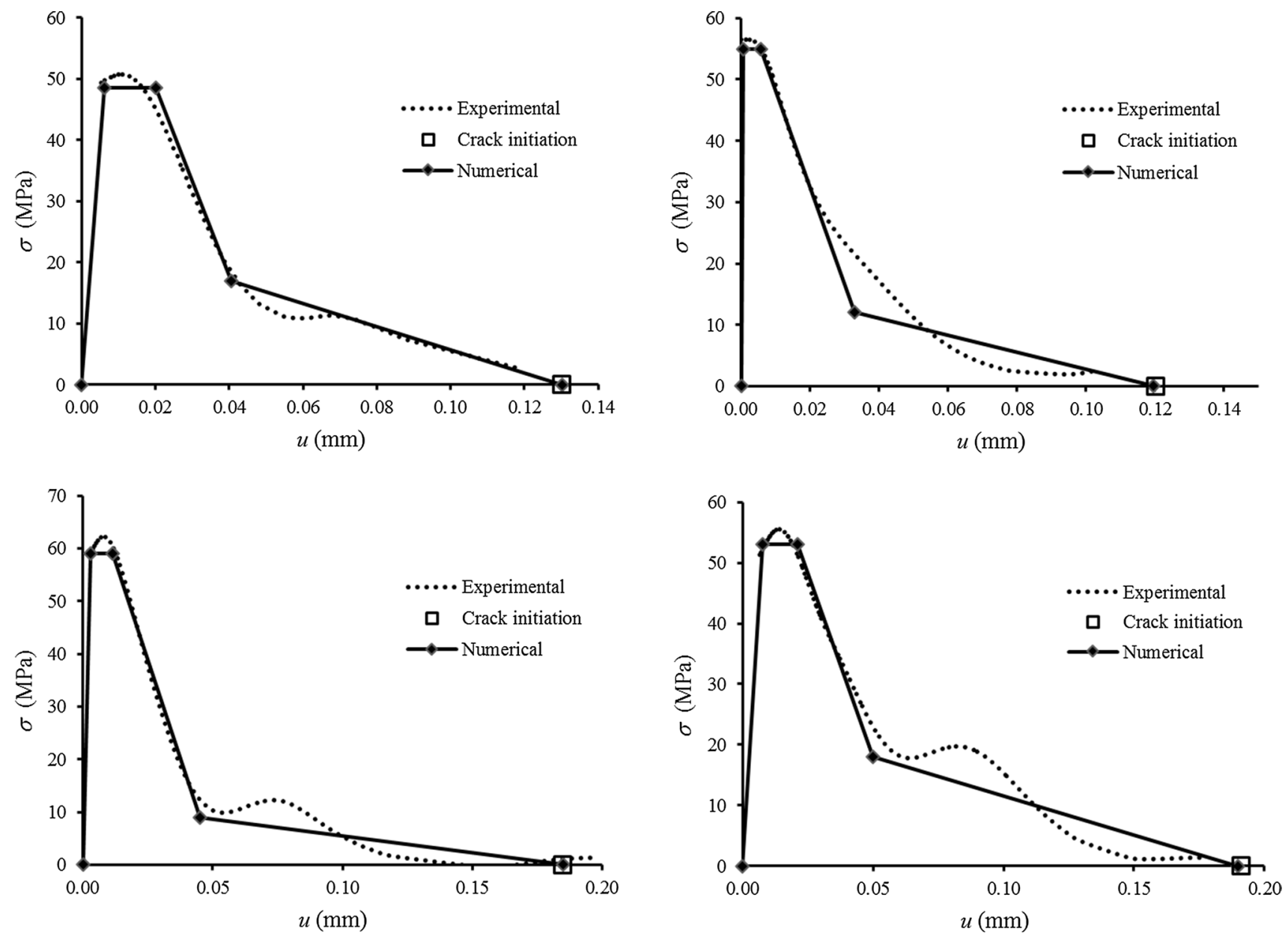

Fig. 8 Experimental and adjusted trapezoidal with bilinear softening cohesive laws

human cortical bone a typical nonlinear fracture mechanics problem.

The four valid load-displacement curves are presented in Fig. 6. Typically, the curves present a large initial linear part, followed by a limited nonlinear region before the peak load which corresponds to the development of the fracture process zone (FPZ). The crack advance usually occurs slightly after the peak load (see point "Crack initiation" in Figs. 6) which means that a pronounced FPZ has developed. In fact, cohesive zone lengths in the range 7-9 $\mathrm{mm}$ were obtained in the four simulated specimens. The selfsimilar crack growth occurs for a limited range of load decrease in the post-peak region. Effectively, the major drawback of the ENF test is the compressive stresses that develop close to the central loading point that affects the natural development of the FPZ and increases artificially the measured fracture energy.

The corresponding $R$-curves (i.e., $\left.G_{\mathrm{II}}=\mathrm{f}\left(a_{\mathrm{e}}\right)\right)$ are presented in Fig. 7. The initial rising trend corresponds to FPZ development till its critical size is attained. After that, a plateau region arises resulting from self-similar crack growth for a small crack extent, i.e., till the compressive stresses in the vicinity of loading point exert its influence leading to spurious increase in fracture energy.

The evolution of $G_{\text {II }}$ as a function of CTSD (parameter $u$ ) curve was fitted using smoothing spline functions to allow differentiation. A detailed analysis about the influence on the obtained cohesive laws of the smoothing parameter $p$ (Eq. 9) was performed. It was verified that $p=0.9$ leads to suitably smooth CLs (Fig. 8). The observed undulations in the experimental CLs correspond to similar phenomena observed in the load-displacement curves and in the $R$-curves. Characteristically, the laws reveal a small plateau in the vicinity of the ultimate stress and two distinct descending parts, which justify the adjustment using trapezoidal with bilinear softening relations to be considered in the numerical simulations (Fig. 8).

\subsection{Numerical}

The numerical analysis was performed to show the appropriateness of the proposed experimental methodologies in 
the context of human cortical bone fracture characterization under mode II loading. The main goal was to verify whether the trapezoidal with bilinear softening CLs fitted to the experimentally measured ones provide good agreement between the numerical and the corresponding experimental results for the load-displacement curve, $R$-curve and CTSD-displacement plotting. The numerical analysis is then fundamental to assure that all the experimental procedure is providing accurate results. Regarding the load-displacement relation, Fig. 6 reveals excellent agreement between the numerical and experimental curves, which constitutes a symptom that the fracture process is being well managed by the fitted cohesive law. The numerical $R$-curves also present good agreement with the experimental ones in the plateau region (Fig. 7), although some discrepancies can be identified at their beginning. This can be explained by some differences between the numerical and experimental initial specimen compliances in the early stages of loading due to specimen
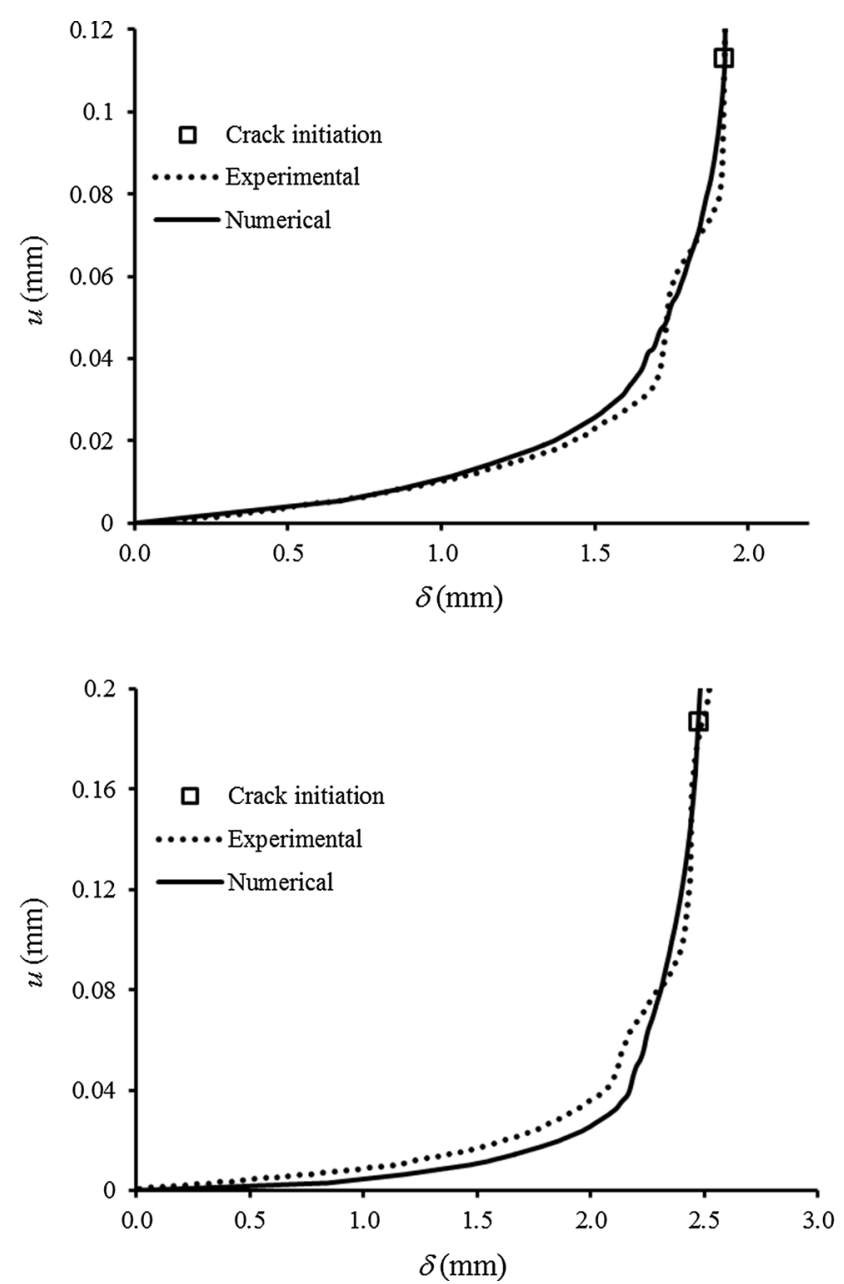

Fig. 9 Experimental and numerical CTSD of the ENF tests accommodation to the supports. In fact, the specimen compliance is the key parameter to obtain the $G_{\mathrm{II}}=\mathrm{f}\left(a_{\mathrm{e}}\right)$ curves in the applied data reduction scheme. In the plateau regions where the fracture energy under self-similar crack growth conditions is defined, excellent agreement is found.

The good agreement observed in Figs. 6 and 7 validates the proposed procedure concerning global parameters, i.e., parameters involving the mechanical behavior of all the specimen, as is the case of load-displacement curves and $R$-curves. In order to verify whether the local parameter CTSD is also well monitored by DIC, the numerical and experimental evolutions of $u=\mathrm{f}(\delta)$ were compared. Numerically, the measurement of CSTD consists of registering the relative displacement at the integration point located at the crack tip, from the beginning of the test till the complete failure at this point has occurred. As shown in Fig. 9, excellent agreement was attained revealing the appropriateness of the procedure.
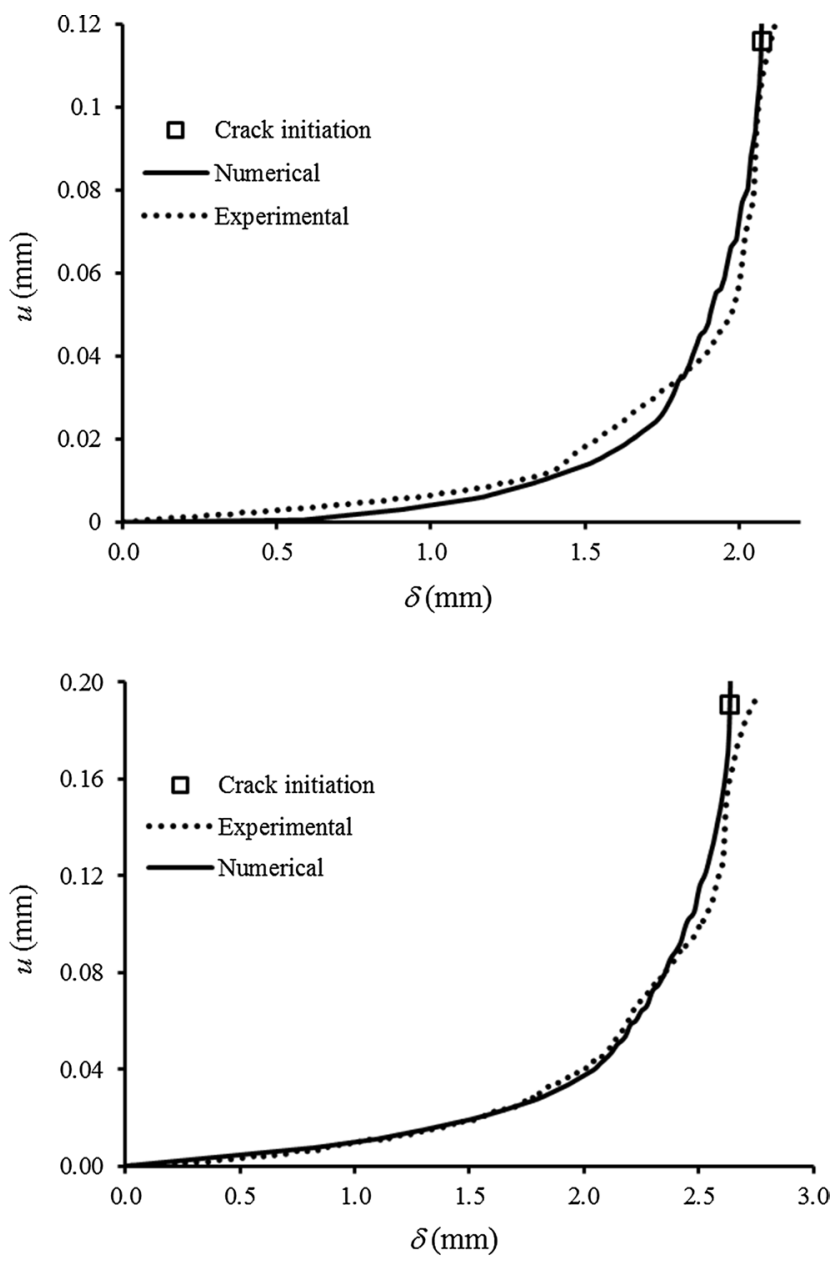


\section{Conclusions}

The application of a miniaturized version of the end-notched flexure test to human cortical bone fracture characterization under mode II loading was analyzed. An equivalent crack length data reduction scheme was used to overcome the difficulties inherent to crack length monitoring. The crack tip shear displacement was measured in the course of the test employing digital image correlation in order to obtain the cohesive laws mimicking fracture process of bone under mode II loading. These procedures aimed two main objectives: (a) to verify whether the ENF test is appropriate for fracture characterization of human cortical bone under mode II loading and (b) to propose a suitable direct method to evaluate the cohesive law characterizing fracture of human cortical bone under mode II loading.

A numerical analysis involving cohesive zone modeling was also performed to validate all the steps of the proposed procedure. A trapezoidal with bilinear softening cohesive law was adjusted to experimental ones to execute numerical analysis. The numerical load-displacement and resistance curves representing global fracture behavior of the specimen revealed excellent agreement with the experimental ones. In addition, the numerical and experimental evolution of crack tip shear displacement as a function of applied displacement was also compared. The excellent agreement observed confirms the validity of the experimental procedure followed to monitor this local parameter. This methodology is an evolution of the studies described in [3] where the cohesive laws were identified by means of an inverse procedure. In that study, an iterative procedure to identify a trapezoidal shape law imposed a priori was followed. In the present study, the proposed methodology is more sophisticated since it relies on the experimental measurement of the real cohesive law using digital image correlation. Following this methodology, it is not necessary to impose (a priori) a pre-defined cohesive law, which can be viewed as an important advantage. In fact, in materials whose fracture behavior is not well known, as is the case of mode II fracture of cortical bone, the choice for an appropriate cohesive law to be fitted in an inverse procedure can be an inexact and difficult task.

It can be concluded that the proposed novel procedure is valid concerning human cortical bone fracture characterization under mode II loading, since it constitutes a useful tool in the context of systematic clinical studies involving aspects (e.g., age, drugs, diseases, environmental and others) that can influence bone fracture resistance which acts as an indicator of bone health. Additionally, the definition of energy-based fracture laws appropriate for bone is a relevant aspect in the design of internal prosthesis providing predictions of damage onset and its propagation in critical regions.
Acknowledgements The authors acknowledge the Portuguese Foundation for Science and Technology (FCT) for the conceded financial support through the research project PTDC/EME-PME/119093/2010.

\section{References}

1. Brown CU, Yeni YN, Norman TL (2000) Fracture toughness is dependent on bone location - a study of the femoral neck, femoral shaft, and the tibial shaft. J Biomed Mater Res 49:380-389

2. Chapra SC (2011) Applied numerical methods with MATLAB ${ }^{\circledR}$ for engineers and scientists. McGraw-Hill Education

3. Dourado N, Pereira FAM, de Moura MFSF, Morais JJL, Dias MIR (2013) Bone fracture characterization using the end notched flexure test. Mat Sci Eng C Mater 33:405-410

4. Gonçalves JPM, de Moura MFSF, de Castro PMST, Marques AT (2000) Interface element including point-to-surface constraints for three-dimensional problems with damage propagation. Eng Comput 17(1):28-47

5. Hambli R (2013) A quasi-brittle continuum damage finite element model of the human proximal femur based on element deletion. Med Biol Eng Comput 51:219-231

6. Leffler K, Alfredsson KS, Stigh U (2007) Shear behaviour of adhesive layers. Int J Solids Struct 44:530-545

7. Morais JJL, de Moura MFSF, Pereira FAM, Xavier J, Dourado N, Dias MIR, Azevedo JMT (2010) The double cantilever beam test applied to mode I fracture characterization of cortical bone tissue. J Mech Behav Biomed Mater 3:446-453

8. Nalla R, Kinney J, Ritchie R (2003) Mechanistic fracture criteria for the failure of human cortical bone. Nat Mater 2:164-168

9. Norman TL, Vashishth D, Burr DB (1995) Fracture toughness of human bone under tension. J Biomech 28:309-320

10. Norman TL, Nivargikar V, Burr DB (1996) Resistance to crack growth in human cortical bone is greater in shear than in tension. J Biomech 29:1023-1031

11. Pan B, Qian K, Xie H, Asundi A (2009) Two-dimensional digital image correlation for in-plane displacement and strain measurement: a review. Meas Sci Technol 20:062001

12. Pereira FAM, Morais JJL, Dourado N, de Moura MFSF, Dias MIR (2011) Fracture characterization of bone under mode II loading using the end loaded split test. J Mech Behav Biomed 4:1764-1773

13. Pope M, Murphy M (1974) Fracture energy of bone in a shear mode. Med Biol Eng Comput 12:763-767

14. Reinsch C (1967) Smoothing by spline functions. Numer Math 10:177-183

15. Silva F, Morais J, Dourado N, Xavier J, Pereira FAM, de Moura MFSF (2014) Determination of cohesive laws in wood bonded joints under mode II loading using the ENF test. Int $\mathbf{J}$ Adhes Adhes 51:54-61

16. Sousa AMR, Xavier J, Morais JJL, Filipe VMJ, Vaz M (2011) Processing discontinuous displacement fields by a spatio-temporal derivative technique. Opt Laser Eng 49:1402-1412

17. Sousa AMR, Xavier J, Vaz M, Morais JJL, Filipe VMJ (2011) Cross-correlation and differential technique combination to determine displacement fields. Strain 47:87-98

18. Wirtz DC, Schiffers N, Pandorf T, Radermacher K, Weichert D, Forst R (2000) Critical evaluation of known bone material properties to realize anisotropic FE-simulation of the proximal femur. J Biomech 33:1325-1330

19. Xavier J, de Jesus AMP, Morais JJL, Pinto JMT (2012) Stereovision measurements on evaluating the modulus of elasticity of wood by compression tests parallel to the grain. Constr Build Mater 26:207-215 
20. Xavier J, Oliveira J, Monteiro P, Morais JJL, de Moura MFSF (2014) Direct evaluation of cohesive law in mode I of Pinus pinaster by digital image correlation. Exp Mech 54:829-840

21. Xavier J, Oliveira M, Morais J, de Moura MFSF (2014) Determining mode II cohesive law of Pinus pinaster by combining the end-notched flexure test with digital image correlation. Constr Build Mater 71:109-115

22. Xavier J, Fernandes JRA, Frazão O, Morais JJL (2015) Measuring mode I cohesive law of wood bonded joints by combining digital image correlation and fibre Bragg grating sensors. Compos Struct 121:83-89

23. Yang QD, Cox BN, Nalla RK, Ritchie RO (2006) Fracture length scales in human cortical bone: the necessity of nonlinear fracture models. Biomaterials 27:2095-2113

24. Zimmermann EA, Launey ME, Barth HD, Ritchie RO (2009) Mixed-mode fracture of human cortical bone. Biomaterials 30:877-5884

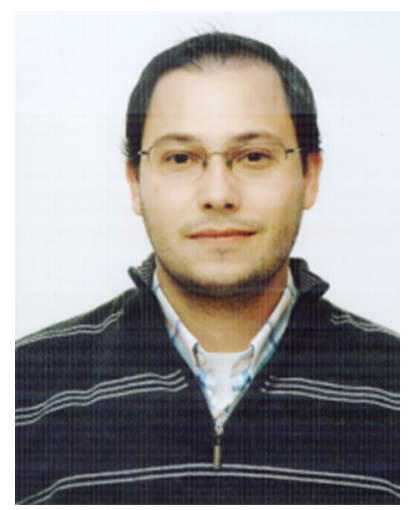

Filipe Silva is currently a $\mathrm{PhD}$ student at Mechanical Department of University of Porto on the subject of fatigue and fracture characterization of cortical bone under pure and mixed-mode loading.

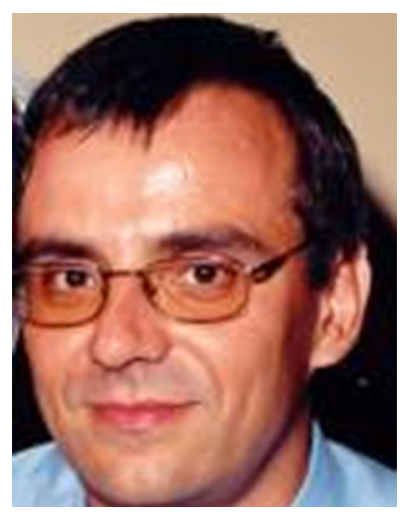

Marcelo Moura is Associate Professor at the Mechanical Department of Faculty of Engineering of University of Porto. He has been working on fatigue/ fracture characterization of anisotropic materials.

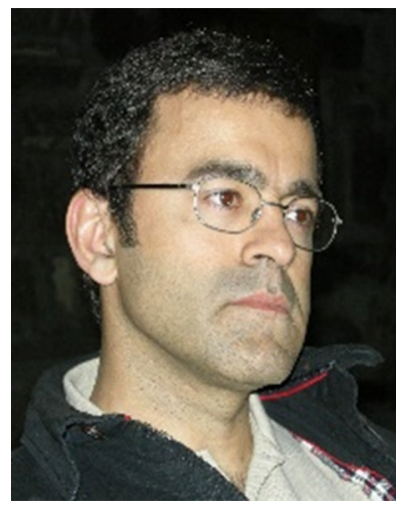

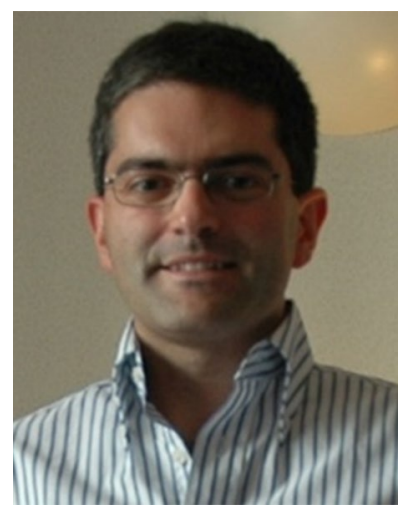

José Xavier completed a $\mathrm{PhD}$ degree in Mechanical Engineering at Arts et Métiers ParisTech in France in 2007. He currently holds a position as invited lecture and research assistant at UTAD/CITAB, PT.

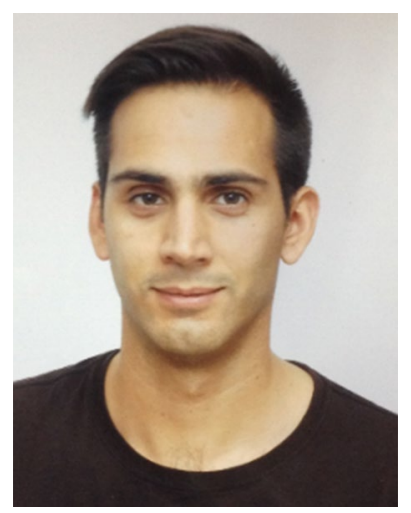

Fábio Pereira is actually an investigator on CITAB with a post doc position. He has been working on fracture characterization of bovine cortical bone under mixed-mode I+II loading.

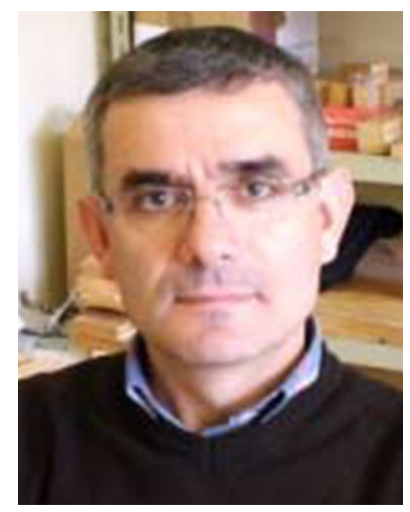

José Morais is full-time professor of Applied Mechanics at the School of Science and Technology of UTAD. His interests are the mechanical and fracture behavior of biological and biobased materials.

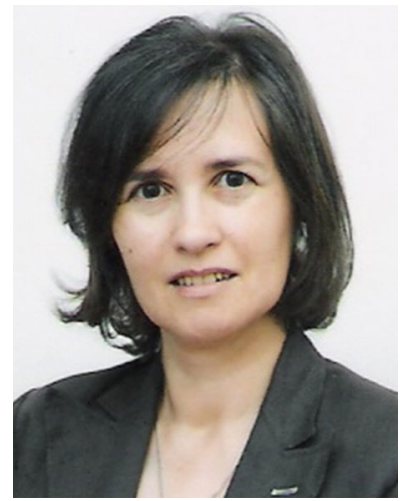

Isabel Dias is Assistant Professor with Habilitation of Small Animal Surgery in the Department of Veterinary Sciences of UTAD. She is actual director of the Teaching Veterinary Hospital. 


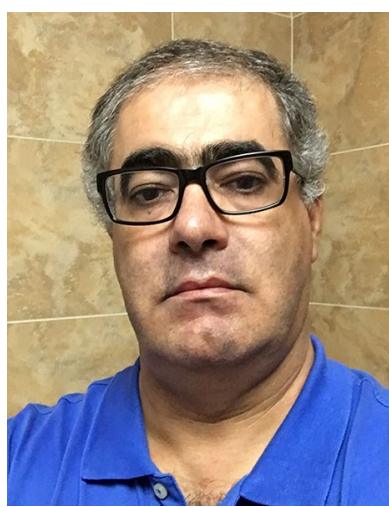

Paulo Lourenço is orthopedic surgeon at the Hospital of the University of Coimbra and Guest Assistant Professor of Biochemistry at the Faculty of Medicine of Coimbra since 1994.

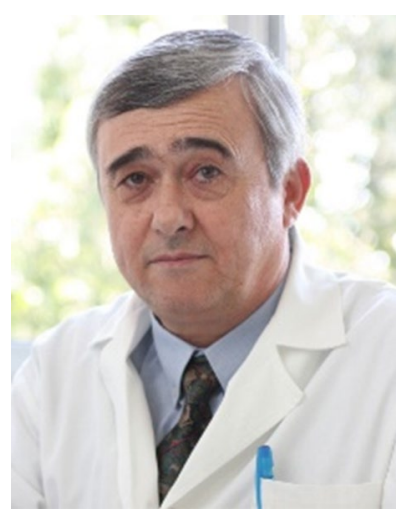

Fernando Judas $\mathrm{MD}, \mathrm{PhD}$, Orthopaedic Surgeon, Chief of Service in the Orthopedics Department, Coimbra Hospital and Universitary Centre, Professor of the Faculty of Medicine, University of Coimbra. 